\title{
Ubiquitous Internet in an Integrated Satellite-Terrestrial Environment: The SUITED Solution
}

\author{
Paolo Conforto, Clementina Tocci, and Giacinto Losquadro, Alenia Spazio, Italy \\ Ray E. Sheriff, Pauline M. L. Chan, and Y. Fun Hu, University of Bradford, United Kingdom
}

\begin{abstract}
The current Internet architecture appears to not be particularly suited to addressing the emerging needs of new classes of users who wish to gain access to multimedia services made available by ISPs, regardless of their location, while in motion and with a guaranteed level of quality.

One of the main objectives of so-called nextgeneration systems is to overcome the limitations of today's available Internet by adopting an approach based on the integration of different mobile and fixed networks. The SUITED project moves in this direction since it aims at contributing to the design and deployment of the global mobile broadband system (GMBS), a unique satellite/terrestrial infrastructure ensuring nomadic users access to Internet services with a negotiated QoS. A description of the main features of the GMBS architecture, characterized by the integration of a multisegment access network with a federated ISP network is given in this article. The GMBS multimode terminal is schematically described, and an overview of the so-called QoS-aware mobility management scheme, devised for such a heterogeneous scenario, is provided.
\end{abstract}

\section{INTRODUCTION}

The Internet protocol architecture was originally conceived with the primary objective of creating a robust and scalable infrastructure, capable of supporting the deployment of a number of applications. Internet users were assumed to be in a steady state, in the office, at home, and so on, and accessing the network by means of wired links. Moreover, the needs of the services and applications requiring simply reliable end-to-end data transfer were satisfactorily supported through the adoption of a best effort service model.
It is now becoming clear that an Internet network with the features described above may not be suitable for addressing the emerging needs of a new population of nomadic mobile Internet users, who desire access to multimedia services regardless of location and mode of transportation. Certainly, the possibility of providing Internet access on land, at sea, or in the air is a highly desirable future service scenario. It is also apparent that the best effort quality of service currently available over the Internet network is becoming increasingly unsatisfactory for several categories of users, who now expect greater variety of services and a more flexible level of service provision to meet their particular requirements.

The growing needs of these new classes of Internet users, who require access to multimedia applications irrespective of location and with the desired quality of service (QoS), are addressed in the framework of the multisegment System for broadband Ubiquitous access to InTErnet services and Demonstrator (SUITED) project. ${ }^{1}$ SUITED aims to contribute to the design and deployment of the Global Mobile Broadband System (GMBS) by focusing, in particular, on its land mobile component. The SUITED GMBS system can be seen as a unique satellite/terrestrial infrastructure obtained by integrating a GMBS multisegment access network with the federated Internet service provider (ISP) network, that is, a part of the (eventually the whole) Internet implementing appropriate functionality. GMBS users provided with suitably designed GMBS multimode terminals (GMMTs) are able to gain access to the multimedia applications an ISP is currently providing, or will be able to provide in the future, by exploiting one of the satellite/terrestrial components in the multisegment access network.

This article aims to provide an overview of 


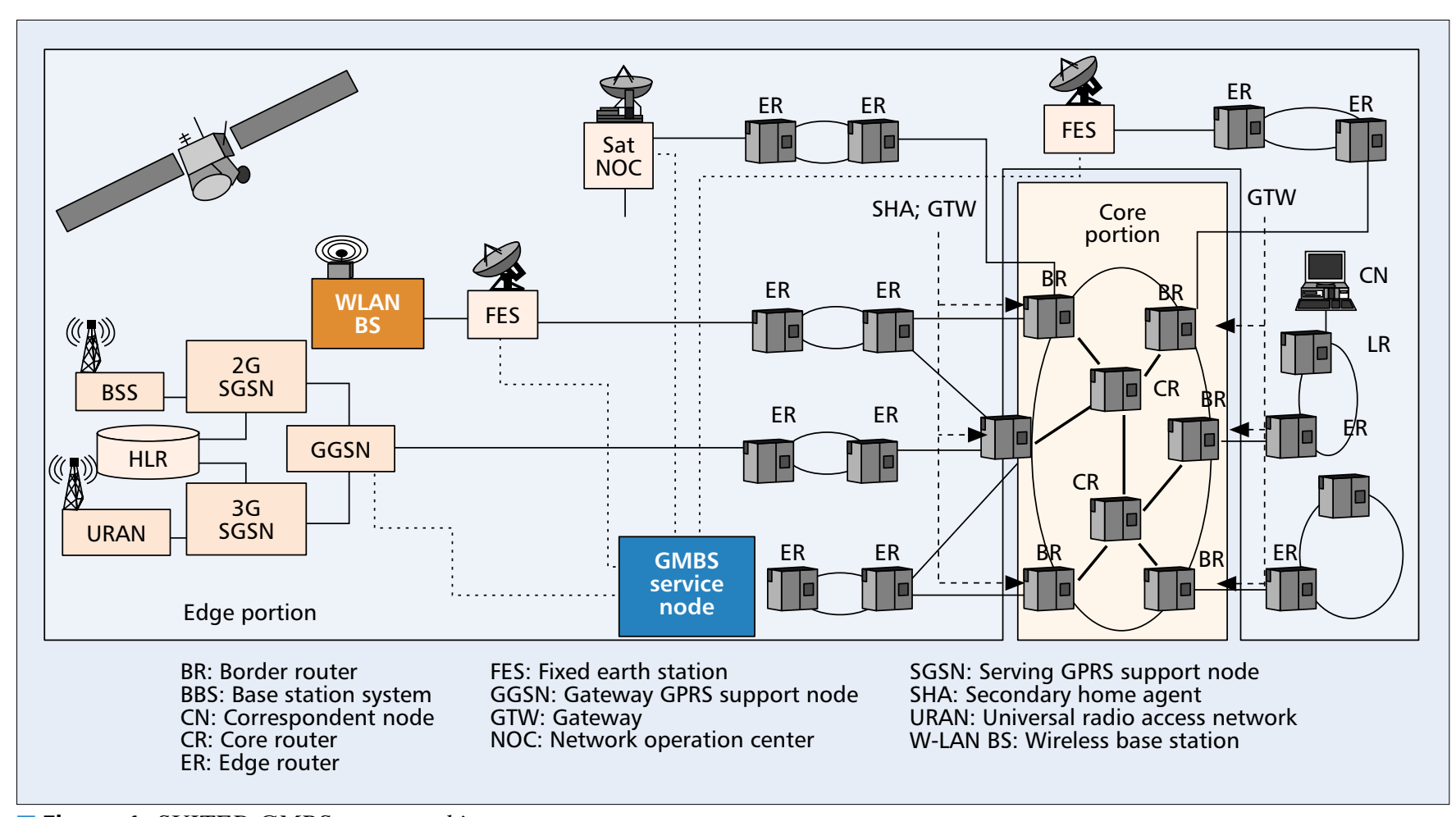

Figure 1. SUITED GMBS system architecture.

the SUITED/GMBS system. The main architectural features are described in the first section. The next section is devoted to the GMMT, which is described in terms of the overall architecture and functional blocks. The approaches followed in designing the global mobility management and QoS support schemes are presented. Lastly, we give some information concerning the SUITED demonstrator, which has been implemented for test and validation purposes.

\section{GMBS SYSTEM ARCHITECTURE}

The service area of the GMBS system shall be worldwide and available in highly diversified operational conditions such as:

- Open/rural environments

- Suburban/urban environments

- Indoor and low-range outdoor environments

Nevertheless, neither wireless terrestrial networks nor satellite systems operating independently are able to serve such a wide range of areas. In order to overcome this issue, the GMBS solution foresees that a multisegment access network, whose components present mutually complementary features, shall interwork with the Internet terrestrial network. From a user perspective, the GMBS system is perceived as a single network capable of supporting mobile and portable QoS-guaranteed Internet services.

The GMBS multisegment access network components are:

- The Ka band onboard processing (OBP) based satellite system

- The General Packet Radio Service (GPRS) system

- The Universal Mobile Telecommunications System (UMTS)

- The wireless LAN (WLAN) system
An evolutionary scenario for the GMBS deployment can be envisaged, whereby in GMBS Phase I (ready to be operational by 2004), the satellite segment will be complemented by the GPRS system, while the GMBS Phase II (ready to be operational by 2006) will be characterized by the presence of the UMTS segment complementing/replacing the GPRS with the task of providing a complementary service coverage area to that of the satellite system. The WLAN will be present in both phases.

An evolutionary scenario is also envisaged for the Internet network within the GMBS system. In the initial phase, Mobile IPv6 and QoS support functionality required for the appropriate integration in the GMBS system are assumed to be present only in a bounded portion of the overall Internet. Such a portion, referred to as federated ISP network, consists of a set of ISPs which have defined peer service-level agreements (SLAs) to provide mobile QoS-sensitive Internet services to a common subscriber population. Subsequent phases will see the extension of the federated ISP network functionality to the remainder of the Internet. The overall GMBS system architecture is schematically depicted in Fig. 1.

The GMBS system is operated by a $G M B S$ service provider (GSP), whose main objective is to provide nomadic GMBS users with connectivity to the Internet network with a guaranteed QoS, irrespective of their position. The GSP controls a GMBS service node $(S N)$, located at the network side, that assists the GMMT in performing several tasks including:

- GMBS user authentication and registration

- Location management

- Transmission of access segment resource allocation information and so on to the GMMT 


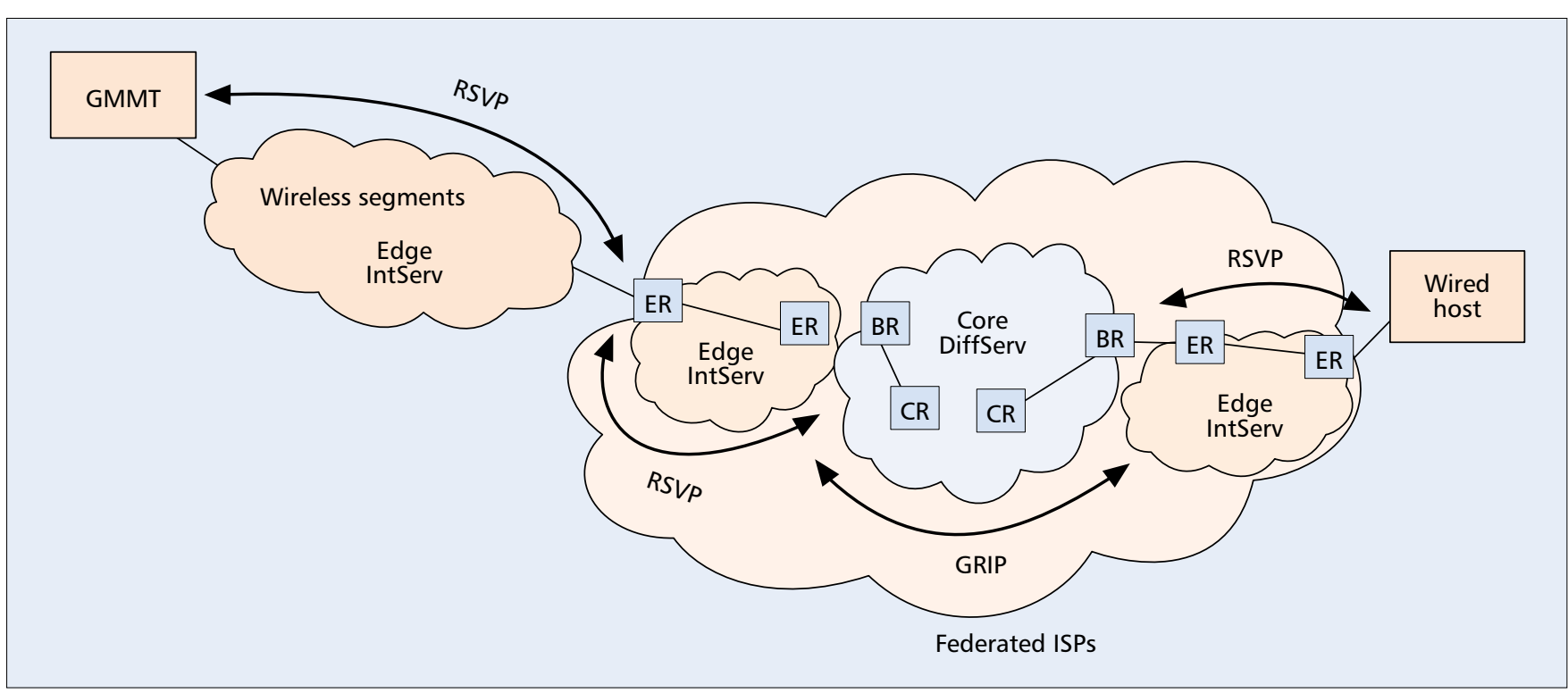

Figure 2. The edge and core portions of the overall GMBS system.

The overall system can be seen to consist of two different portions (Fig. 2):

- An edge portion: consisting of the wireless segments and the Internet edge terrestrial subnetworks

- A core portion: consisting of one or more Internet subnetworks and, optionally, of a satellite segment (acting as transit ISP)

An integrated services (IntServ) model based on the Resource Reservation Protocol (RSVP) [1, $2]$ is adopted in the edge portion, while a differentiated services (DiffServ) model, enhanced with a measurement-based admission control mechanism named Gauge and Gate Reservation with Independent Probing (GRIP) [3] explicitly devised in the framework of SUITED, is implemented in the core portion. As far as the mobility aspects are concerned, Mobile IPv6 [4] is implemented in the federated ISP network.

\section{GMBS Multi-Segment Access Network}

The systems (also referred to as segments) that forms the GMBS multisegment access network are as follows:

The Mobile EuroSkyWay (M-ESW) broadband satellite system is currently being devised to offer connectivity services to several different heterogeneous terrestrial networks by means of a number of Ka-band geostationary satellites with OBP functionality. The M-ESW acts as an underlying network with respect to the existing terrestrial networks to which it is connected. In fact, M-ESW provides the means to transparently transfer both signaling and traffic data generated both at the terminal level and by external networks, which for this reason can be logically considered as overlaying networks. This is realized by implementing a minimum set of protocols and signaling procedures that are applicable to the satellite system. These protocols are used to efficiently control the provision of switched capacity on demand and for mobility/localization purposes. As depicted in Fig. 1, M-ESW connects to the Internet network through multiple points of attachment, each represented by a fixed Earth station (FES). A network interface unit (NIU), located at each FES, allows interworking between the IP protocol and the MESW-specific protocols. The satellite network operation center (NOC) represents a centralized entity, which is in charge of executing synchronization, registration, authentication and connection admission control procedures. Moreover, the NOC is also in charge of selecting, according to appropriate algorithms, the most suitable FES to support data traffic and for assigning IP addresses to the terminals.

The GPRS system provides a new bearer service allowing efficient wireless access to packet data networks (e.g., the Internet). It uses a packet mode technique to transfer high speed and low speed data and signaling in an efficient way between mobile stations and external data networks.

UMTS allows bit rates ranging from $144 \mathrm{~kb} / \mathrm{s}$ to $2 \mathrm{Mb} / \mathrm{s}$ depending on the environment (i.e., rural, suburban, or indoor/low range) and the maximum speed of the user.

A $W L A N$ system is considered to provide the mobile with user backup access to the satellite segment. It aims to prolong the satellite link in environments such as low-range outdoor and indoor, where satellite link availability is very poor or not present, by connecting a WLAN base station (BS) to the M-ESW FES. The WLAN implementation is based on the IEEE 802.11 standard.

Each system in the GMBS multisegment access network is able to assign one or more IP addresses to a mobile router (i.e., a GMMT), which connects to the Internet via the wireless link provided by the access segment. The IP address(es) assigned is (are) interpreted as careof address(es) (CoA) of the mobile router. As far as QoS support over the GMBS multisegment access network is concerned, since an IntServ model based on RSVP is adopted in the edge portion of the GMBS, suitable interworking functionality is needed to allow interworking between the access segment-specific protocols and RSVP. Such an interworking functionality is 
implemented in a QoS support module (QASM) hosted at both the terminal and network sides, in appropriate access segment nodes (i.e., the FES and GGSN).

\section{The Federated ISP AND INTERnet Network}

The federated Internet service provider (ISP) network represents a bounded portion of the Internet network, where both Mobile IPv6 and QoS support functionality required for the appropriate integration in the GMBS system are implemented. In subsequent phases of the GMBS evolutionary deployment, such functionality shall be extended to the rest of the Internet (or at least to other significant portions of the network).

The federated ISP architecture can be considered as a peer-to-peer environment, where ISPs are connected through network access points (NAPs) or direct interconnections. According to the structure of the overall GMBS system, the federated ISP network is partitioned into an edge network and a core network. The edge network is the point at which local mobile users are allowed into the network. By adopting the IntServ model in the edge network, RSVP is employed by users to request specific QoS from the network, and by routers to set and maintain a state to provide the requested service with hard QoS guarantees. Nevertheless, the RSVP approach, due to its per-flow orientation and state maintenance within the network, is not scalable; therefore, its usage is precluded in large-scale networks such as the high-speed backbones of ISPs. For this reason, the scalable DiffServ model is adopted in the federated ISP core network. Moreover, since DiffServ does not provide strict end-to- end QoS assurances, an innovative solution developed in the framework of SUITED, GRIP [3], is implemented in the core portion in order to improve user-perceived QoS performance. GRIP is a fully distributed and scalable admission control scheme that allows hard QoS guarantees to be achieved over a stateless DiffServ Internet. The interoperation between IntServ and enhanced DiffServ mechanisms takes place within suitably devised gateways collocated within border routers (i.e., routers located at the interface between the core and the edge portions). Such interoperation requires the implementation of IntServ/DiffServ service mapping and activation of the GRIP distributed admission control mechanism, which takes into account the resource availability in the core network.

The hybrid Intserv/Diffserv solution devised for the SUITED system is particularly attractive since it:

- Solves scalability problems (DiffServ in the core portion)

- Provides a means to easily adapt to the future evolutions of the Internet network (toward completely DiffServ or IntServ solutions)

- Allows the configuration of a fully IntServcompatible end-to-end path in instances where only the edge portion is involved

As far as mobility support is concerned, Mobile IPv6 is implemented in the edge part of the federated ISP network. In order to manage a QoS-aware intersegment handover (ISHO) so that it is executed seamlessly when a change in the supporting access segment takes place, a new mobility agent devised in the framework of SUITED [5] and named secondary home agent $(S H A)$ is introduced at the point of attachment to the Internet. The SHA supports, on the network side, the service that allows QoS IP connectivity to be transparent to mobility issues. The SHA is simply a software module that is fully integrated with resource management and mobility support modules, and runs in suitably selected routers. Adopting an evolutionary approach to the deployment of the SHAs, a first phase may be characterized by their integration in border routers, while subsequent phases may envisage a hierarchical structure with SHAs progressively added in routers closer to the edge of the network. The SHA is in charge of:

- Managing the mobility of GMMT by adopting a regional/hierarchical registration-like approach $[6,7]$ so that handover can be transparent to both the correspondent node and home agent of the terminal equipment (TE) (Table 1)

- Performing both an availability test and reservation of the resources on the new path before switching the IP packet flow by executing RSVP message duplication/selection operations

\section{The GMBS MuLtimode TeRMinal}

GMMTs have been devised for both individual and collective use in three different typologies:

- Car version terminal

- Large vehicle (e.g., trains, buses, ships) version terminal

- Briefcase version terminal

The GMMT internal structure is schematically depicted in Fig. 3, while Table 1 lists its main blocks, describing the functionality for which they are responsible.

The data rates of the different segment-specific terminals operating in both the individual and collective configurations are summarized in Table 2 and classified on the basis of mobility features and operational environments. Moreover, the service area associated with each segment-specific terminal is also indicated.

\section{TERMINAL INTER-WORKING UNIT}

In the heterogeneous environment of the SUITED/GMBS system, which is characterized by an access network consisting of several different satellite/terrestrial segments, a significant amount of the coordination activity is assigned to the GMMT. To this end, the T-IWU, hosting SUITED-specific entities, plays a central role since it is involved in all the intersegment mobility procedures defined in SUITED and cooperates with the access-segment-specific mechanisms for the provision of QoS over the GMBS multisegment access network. The T-IWU functional block diagram is depicted in Fig. 4. Each block represents a software module, grouping similar functionality executed inside the T-IWU. The T-IWU modules are described in the following.

The QoS support module is in charge of:

- Performing interworking between the access-segment-specific protocols and
The RSVP

approach, due to

its per-flow

orientation

and state

maintenance

within the

network, is not

scalable;

therefore, its

usage is precluded

in large-scale

networks such as

the high-speed

backbones of

ISPs. For this

reason, the

scalable DiffServ

model is adopted

in the federated

ISP core network. 
Broadband satellite terminal (SaT)

GPRS mobile terminal (MT)

UMTS mobile terminal (MT)

WLAN mobile terminal (MT)

Terminal interworking unit (T-IWU)

Terminal equipment (TE)

GMBS smart card

Navigation unit

Satellite antenna

P implemented to support an IntServ model on the GMBS edge portion

- Executing functions (e.g., traffic shaping, policing, classification, scheduling, and congestion control) aiming to complement the access-segment-specific mechanisms for the provision of QoS so as to render the GMBS multisegment access network with a unique and uniform interface for the QoS provision from the IP perspective.

The enhanced Mobile IP module implements Mobile IP (MIP) functionality with enhanced capability to support a QoS-aware ISHO procedure.

The registration handler module hosts functionality mainly in charge of dealing with the execution of registration and authentication procedures. Such procedures occur at two different levels:

- At the access segment level (i.e., segmentspecific terminal registration procedure)

- At the GMBS system level (i.e., GMBS user registration and authentication procedure)

The location handler module executes the procedures needed to locate user terminals and establish IP connectivity. In particular, this module is in charge of:

- Executing intersegment mobility procedures such as segment selection/reselection, which results in the definition of the access segment to be used to support the IP data traffic

- Interacting with the segment-specific terminal in order to trigger segment-specific procedures, such as satellite connection setup or PDP context activation in order to establish wireless connectivity

The handover handler module is in charge of dealing with the ISHO procedure. Three different phases are envisaged:

- Handover information gathering

- Handover decision

- Handover execution

The correct execution of the procedures of which the T-IWU is in charge requires strict interaction among the different modules. Such interactions take place by means of suitably devised commands used to trigger internal routines and to pass the necessary parameters. These commands are schematically indicated in Fig. 4 by arrows linking the different modules. Bold arrows are used to represent the flow of IP packets.

The interaction between the T-IWU and the segment-specific mobile terminals (SS-MTs) (i.e., the M-ESW SaT, GPRS/UMTS MT, and WLAN MT) is performed through two service access points (SAPs): 


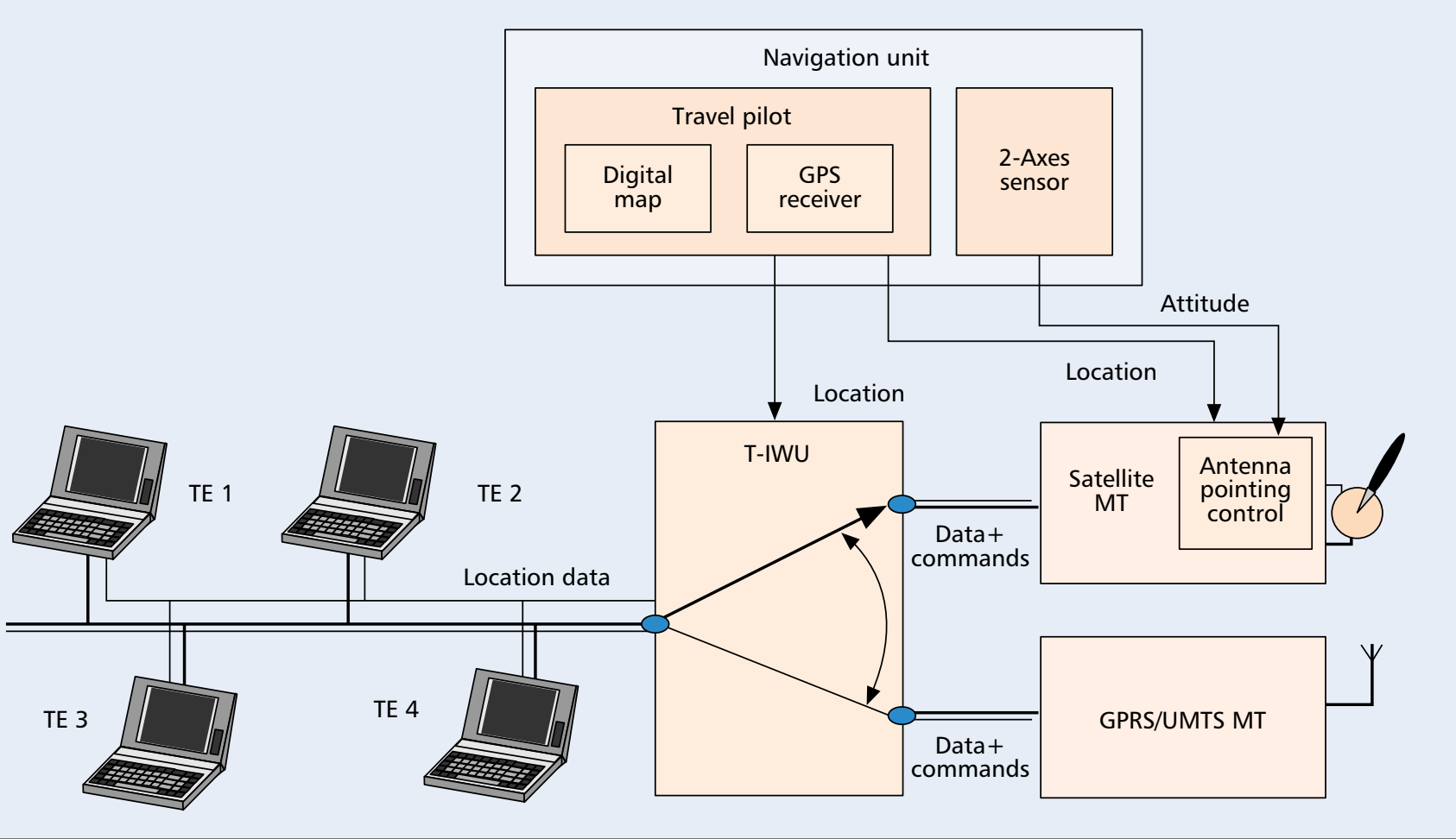

Figure 3. GMMT internal structure.

- SAP for data transfer where the IP packets go through

- $S A P$ for commands through which specific commands (e.g., AT commands in the case of GPRS/UMTS terminals) are exchanged in order to trigger segment-specific procedures

\section{A QOS-AWARE Mobility MANAgement SCHEME}

Global mobility management and end-to-end QoS support are strictly related to each other. In the framework of the SUITED/GMBS system, in order to highlight their close interaction, it is preferable to adopt the term QoS-aware mobility management scheme. In this section an overview of this scheme is provided.

\section{Mobility Management}

In order to support global mobility, an appropriate GMBS mobility management (MM) scheme has been devised. Considering the main architectural features of the system, the GMBS MM scheme derives from the harmonization of the following three levels of mobility management: - IP mobility management: This is based on MIP implemented in the Internet network.

\begin{tabular}{|c|c|c|c|c|c|c|}
\hline & & \multicolumn{3}{|c|}{ Mobile } & \multirow[t]{2}{*}{ Portable } & \multirow[t]{2}{*}{ Service area } \\
\hline & & $<10 \mathrm{~km} / \mathrm{h}$ & $<120 \mathrm{~km} / \mathrm{h}$ & $<250 \mathrm{~km} / \mathrm{h}$ & & \\
\hline \multirow[t]{2}{*}{ Satellite } & Individual & $\begin{array}{l}\text { Uplink: } 160 \mathrm{~kb} / \mathrm{s} \\
\text { Dwlink: } 6 \mathrm{Mb} / \mathrm{s}\end{array}$ & $\begin{array}{l}\text { Uplink: } 160 \mathrm{~kb} / \mathrm{s} \\
\text { Dwlink: } 6 \mathrm{Mb} / \mathrm{s}\end{array}$ & N/A & $\begin{array}{l}\text { Uplink: } 160 \mathrm{~kb} / \mathrm{s} \\
\text { Dwlink: } 16 \mathrm{Mb} / \mathrm{s}\end{array}$ & $\begin{array}{l}\text { Rural, } \\
\text { suburban/urban }\end{array}$ \\
\hline & Collective & $\begin{array}{l}\text { Uplink: } 0.512 / 2 \mathrm{Mb} / \mathrm{s} \\
\text { Dwlink: } 16 \mathrm{Mb} / \mathrm{s}\end{array}$ & $\begin{array}{l}\text { Uplink: } 0.512 \text { / } 2 \mathrm{Mb} / \mathrm{s} \\
\text { Dwlink: } 16 \mathrm{Mb} / \mathrm{s}\end{array}$ & $\begin{array}{l}\text { Uplink: } 2 \mathrm{Mb} / \mathrm{s} \\
\text { Dwlink: } 16 \mathrm{Mb} / \mathrm{s}\end{array}$ & $\begin{array}{l}\text { Uplink: } 0.512 \text { / } 2 \mathrm{Mb} / \mathrm{s} \\
\text { Dwlink: } 16 \mathrm{Mb} / \mathrm{s}\end{array}$ & $\begin{array}{l}\text { Low-range outdoor/ } \\
\text { indoor (via WLAN), } \\
\text { Polar zones (via LEO) }\end{array}$ \\
\hline GPRS & Collective & $N \quad 150 \mathrm{~kb} / \mathrm{s}$ & $N \quad 150 \mathrm{~kb} / \mathrm{s}$ & $N \quad 150 \mathrm{~kb} / \mathrm{s}$ & $N 150 \mathrm{~kb} / \mathrm{s}$ & $\begin{array}{l}\text { Low-range outdoor/ } \\
\text { indoor }\end{array}$ \\
\hline \multirow[t]{2}{*}{ UMTS } & Individual & $2000 \mathrm{~kb} / \mathrm{s}$ & 384 / $512 \mathrm{~kb} / \mathrm{s}$ & $144 / 384 \mathrm{~kb} / \mathrm{s}$ & $2000 \mathrm{~kb} / \mathrm{s}$ & Suburban/urban \\
\hline & Collective & $N \quad 2000 \mathrm{~kb} / \mathrm{s}$ & $N \quad 384 / 512 \mathrm{~kb} / \mathrm{s}$ & $N \quad 144 / 384 \mathrm{~kb} / \mathrm{s}$ & $N \quad 2000 \mathrm{~kb} / \mathrm{s}$ & $\begin{array}{l}\text { Low-range outdoor/ } \\
\text { indoor }\end{array}$ \\
\hline
\end{tabular}

* The WLAN is used as prolongation of satellite connectivity.

Table 2. Segment-specific terminal data rates and service areas. 


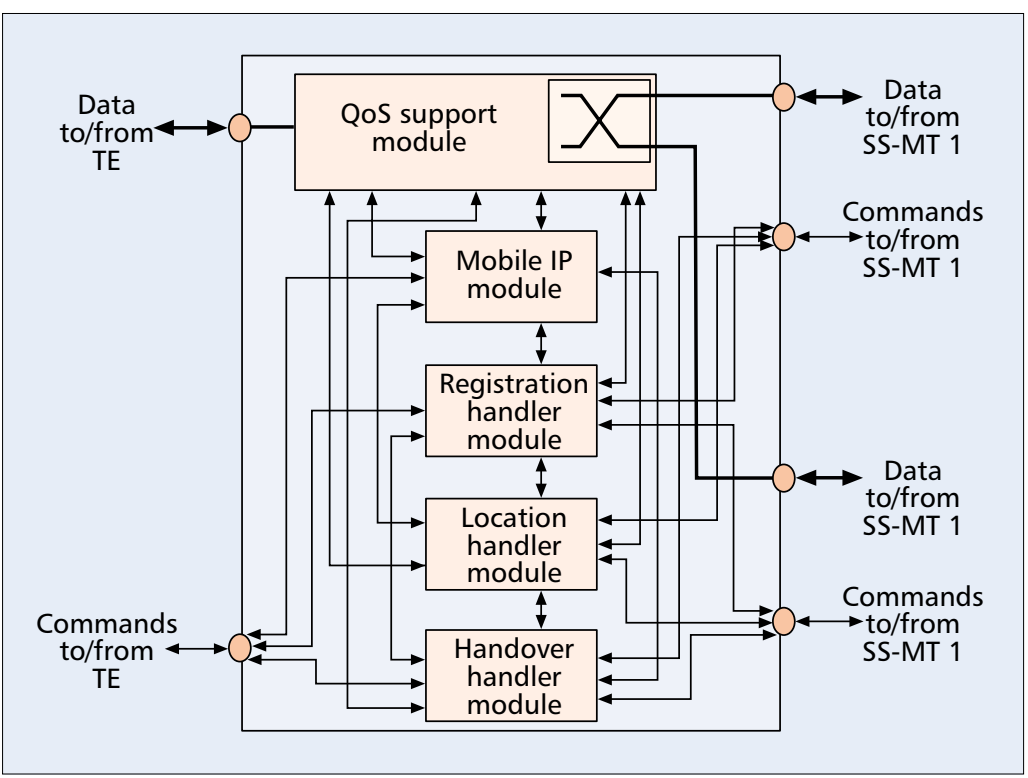

Figure 4. T-IWU functional block diagram.

Mobile IPv6 has been considered the baseline even though full compatibility with Mobile IPv4 is also taken into account.

- Intersegment mobility management: This is devised in order to allow the GMBS user to move from one access segment to another.

- Intrasegment mobility management: This is completely handled by the entities of the access segment serving the GMMT at a particular time, as long as the GMMT remains within its radio coverage.

It is worth highlighting that the class of intrasegment mobility management consists of all the procedures already specified in each access segment (i.e., M-ESW and GPRS/UMTS). No modifications are envisaged for such segment-specific procedures apart from the two cases represented by:

- Intrasegment handover for low-range outdoor/indoor coverage; this is explicitly devised in the framework of the SUITED project to exploit the WLAN segment as a prolongation of the satellite connection in shadowed/indoor areas, where the satellite link is poor or unavailable

- Smart routing, executed at satellite connection setup in order to select the most suitable FES; this procedure allows meaningful improvement of the end-to-end QoS, since the adopted selection criterion is based on reduction of the terrestrial path between the edge router connected to the FES and the correspondent node involved in the application

A generic GMBS user provided with a GMMT is able to connect to each of the multisegment access network components. The GMMT can be seen, from the Internet point of view, as a mobile node [4] (or more precisely a mobile router ${ }^{2}$ ). A mobile node can change its point of attachment from one link to another, while still being reachable via its home address. In order to access the Internet network, the mobile node represented by the GMMT can select one of the access components that, according to the results of an appropriate algorithm, is "the most suitable one." The point of access to the Internet will vary, depending on the component chosen to support IP packet transmission.

The GMMT, or more precisely the T-IWU, is assigned a (set of) different IP address(es) depending on the selected segment. A change in access segment (intersegment mobility) implies a change of CoA (IP mobility). As long as the GMMT remains in the same access segment, no change in the $\mathrm{CoA}$ is required, and the mobility is completely managed by the access-segmentspecific entities (intrasegment mobility). To further elaborate on the approach to $\mathrm{CoA}$ assignment, consider the case of a collective terminal where several TEs - each corresponding to a GMBS user - are connected through a LAN to the same T-IWU (Fig. 3). The T-IWU assigns a CoA to each TE (i.e., the $\mathrm{COA}_{\mathrm{TE}}$ ). Moreover, the T-IWU is assigned a different CoA (i.e., $\mathrm{CoA}_{\mathrm{T}-\mathrm{IWU}}$ ), dependent on the selected segment. This CoA, assigned by the GGSN or ESW NOC in the case of GPRS/UMTS or ESW segment selection, respectively, is used to route the IP packets, within the federated Internet network, toward the correct GGSN or FES. As soon as the GGSN or FES receives these IP packets, the routing toward the mobile router is in charge of the access-segment-specific entities.

The GMBS mobility management scheme is based on the execution of procedures belonging to three different classes:

GMBS generic procedures: This class is composed by the set of registration and authentication procedures. Such procedures take place at two different levels:

- At the segment-specific level - GMBS

(de)registration and authentication

- GMBS level - GMBS user (de-)registra-

tion and authentication

GMBS location management procedures: All procedures needed to locate users and terminals. Five different procedures are envisaged:

- GMBS location update procedure

- Segment selection procedure

- Segment reselection procedure

- GMBS session establishment procedure

- GMBS session release procedure

GMBS handover management procedures: This makes up the ISHO procedure, which is executed in order to change access segment during an active IP session; one of the main requirements to be satisfied is that the QoS perceived by the user should not suffer significant degradation due to such a change. Two different kinds of ISHO can be executed:

- Forced handover, which takes place when a sudden drop in link layer QoS occurs (e.g., the radio link is lost due to fading or interference)

- QoS-aware handover, which is executed when "old" and "new" access segments are simultaneously available for a short time to seamlessly switch the point of attachment to the Internet without any reduction in IP QoS

\section{END-TO-END QOS SUPPORT}

In recent years, work performed in the framework of the Internet Engineering Task Force (IETF) standardization body to provide the Internet with QoS support capability, has led to two distinct approaches: 
- The IntServ model with the relevant RSVP $[1,2]$

- The DiffServ model [8]

One of the most challenging objectives of the SUITED GMBS system is to support Internet QoS-sensitive services in order to provide users with end-to-end QoS guarantees. When an application is started in the TE, a GMBS session establishment (see previous section) is triggered in order to "set up" an IP connection ${ }^{3}$ in charge of supporting the associated data traffic. According to the main characteristics of the GMBS system architecture, in the most general case this connection involves different network portions:

- An edge portion consisting of wireless segments and edge terrestrial subnetworks where an IntServ model is adopted

- A core portion, consisting of one or more terrestrial subnetworks, characterized by a DiffServ model (optionally a satellite segment can be included in the core portion, in which case the IntServ approach applies) Considering that the IntServ and DiffServ architectures can be seen as complementary technologies in pursuit of IP end-to-end QoS, the solution adopted in the SUITED system is based on the so-called hybrid IntServ-DiffServ approach (Fig. 2 ). The rationale is to exploit on one side the possibility for the hosts to request quantifiable resources along end-to-end data paths, possibly provided by the IntServ architecture, and on the other the scalability properties provided by the DiffServ architecture.

The establishment of IP connectivity with end-to-end QoS guarantees, between one of the TEs in the GMMT and a wired host, ${ }^{4}$ requires that an RSVP session be activated for each direction of communication (i.e., TE toward wired host and wired host toward TE).

The followed approach foresees that, in the deployment of an RSVP session, both the wireless segments and the DiffServ core portion of the federated ISP network play the role of "network elements" in the IntServ framework and are used as components of an overall endto-end IntServ QoS solution. More precisely, the access portion between the terminal and network $Q A S M$, as well as the core portion between two gateways, are seen, from the RSVP perspective, as simple RSVP-capable routers. This implies that QASM and gateway entities participate in RSVP session execution by updating the Adspec parameters in the RSVP Path message in order to take into account the features of the network portion for which they are responsible, and by evaluating if in the same portion the resources required by the RSVP Resv message are available. It is worth stressing that all RSVP messages exchanged between the TE and the wired host during an RSVP session evolve transparently on the core portion of the federated ISP network. Non-RSVP-capable routers do not affect these messages, since RSVP is designed to operate correctly through a non-RSVP cloud.

Such an approach allows the criticality associated with the proposed scenario to be overcome, where heterogeneous systems adopting both IntServ and DiffServ models coexist. Such criticality is represented by two issues:
- IntServ operation over a DiffServ network along with the relevant service mapping

- The mapping between the IntServ/RSVP requests and the underlying capabilities of the SUITED wireless segments

The first issue is solved within the gateways that, placed at the interface between the DiffServ and IntServ region of the federated ISP network, are in charge of executing, at the reception of an RSVP Resv message, the mapping of an IntServ/RSVP request into a DiffServ GRIP class. This is followed by the activation of the GRIP distributed admission control mechanism, which aims to determine whether adequate network resources are actually available to support the requested QoS.

A solution to the second issue is represented by the introduction of the QASM module at both the terminal and network sides. The QASM entities intercept and reconstruct the RSVP Resv messages and then execute the mapping of RSVP parameters onto access segment specific parameters. Once this mapping is executed, the QASM triggers the access segment connection admission control mechanism to verify that available resources exist and, if so, to reserve them.

\section{The SUITED DEMONSTRATOR}

An extensive experimental campaign is envisaged in the framework of the SUITED project to test and validate the main functionality devised for the GMBS system for the support of both mobility and QoS. This section, due to length constraints, aims just to provide some information pertaining to the SUITED prototypal layout designed and implemented in order to validate the IP-based infrastructure devised for the provision of mobile QoS-sensitive Internet services.

Figure 5 shows the SUITED demonstrator layout whose main entities are:

- The multimode terminal (based on a T-IWU, embedded on a PC/104 Plus, 32-bit PCI bus onboard system, interfacing on one side the user TE and on the other the wireless access segments) installed into the vehicle

- The regenerative satellite segment simulated via the operating Italsat satellite and enhanced with a laboratory model of the regenerative stage

- The GPRS/UMTS and WLAN access segments

- The federated ISP network represented by two core routers (CRs) interfaced with two edge routers (ERs)

The ERs are a 3640 Cisco router and a 2611 Cisco router. The CRs are PCs based on Linux and provided with a suitable custom software to manage the DiffServ method upgraded with the GRIP measurement-based admission control mechanism.

The RSVP session is started by the user. The RSVP messages are analyzed by both the satellite network to establish satellite connections with the proper characteristics and the ERs to reserve the appropriate bandwidth. The interaction between the IntServ/RSVP and DiffServ models is realized in the access router (AR), where the RSVP packets are analyzed to extract the information about the required QoS. This information is used to start the GRIP protocol
The followed

approach

foresees that, in

the deployment

of an RSVP

session, both the

wireless segments

and the DiffServ

core portion of

the federated ISPS

network play the

role of "network

elements" in the

IntServ

framework and

are used as

components of

an overall end-to-

end IntServ

QoS solution.

${ }^{3}$ The term IP connection establishment, although sometimes considered not technically correct, is here strictly related to the need to both set up a wireless link supporting the exchange of IP packets and execute an RSVP session for QoS support purposes.

4 Similar considerations can be extended to the case where the connection is between two TEs in two different GMMTs. 


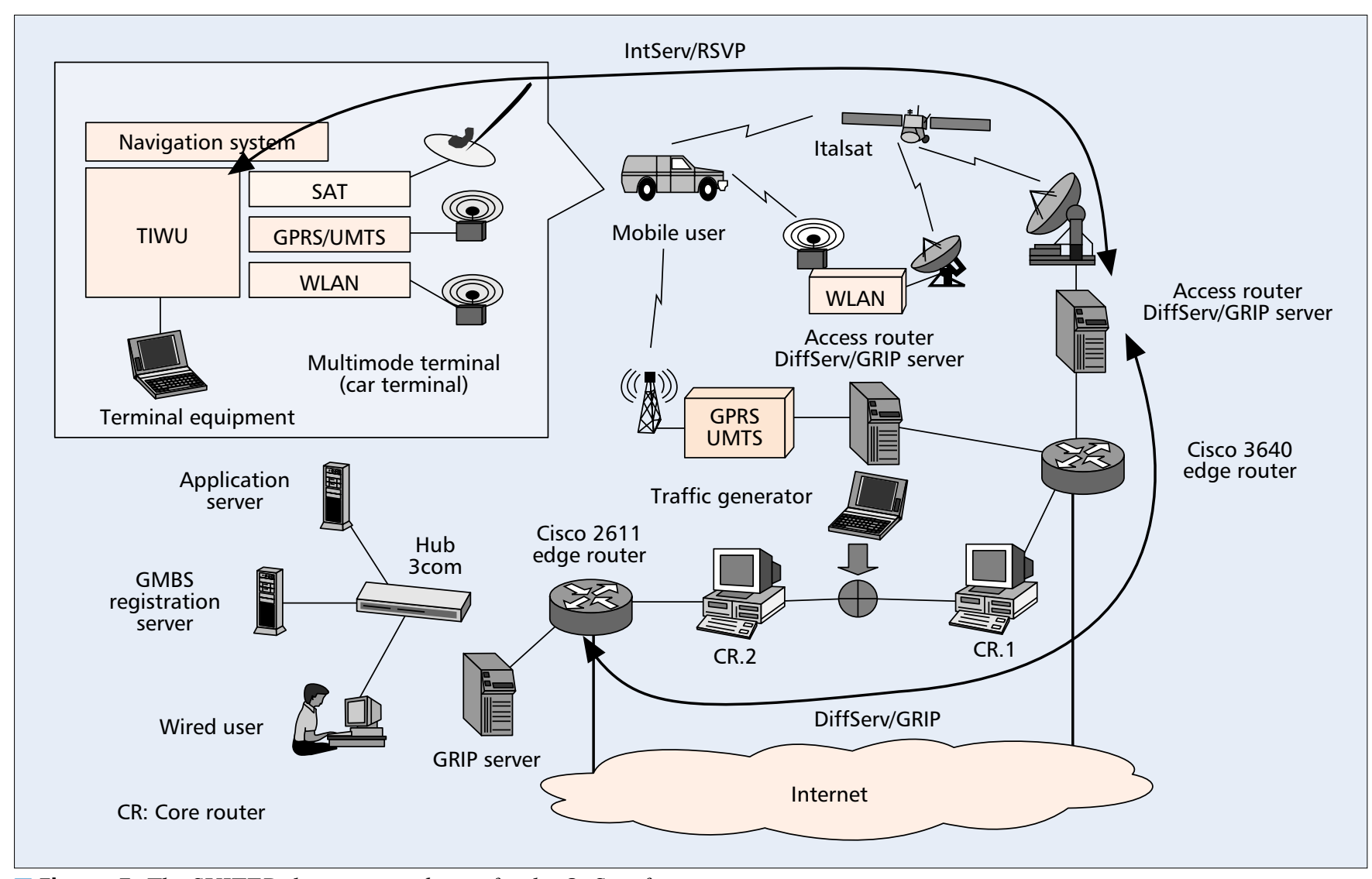

Figure 5. The SUITED demonstrator layout for the QoS performance test.

and modify the user datagrams, setting the proper value in the Type of Service (ToS) fields, to implement the DiffServ model.

To simulate a traffic load between the two CRs a traffic generator is inserted. Then, during the traffic exchange, a protocol analyzer measures and analyzes the links' performance.

\section{CONCLUSIONS}

In this article an overview of the GMBS system developed in the framework of the SUITED project is provided. The system architecture has been described, highlighting the main innovative solutions at both the network and terminal levels.

A multisegment access network consisting of different satellite/terrestrial components is integrated with a federated ISP network to create an infrastructure seen by users as a unique network allowing access to Internet services irrespective of location, while mobile, and with a guaranteed QoS.

A QoS-aware mobility management scheme has been devised in order to support global mobility along with end-to-end QoS.

\section{ACKNOWLEDGMENT}

This work has been partially funded by the European Commission in the IST Program under the SUITED project. The authors wish to thank the members of the SUITED Consortium for their contribution.

\section{REFERENCES}

[1] R. Braden et al., "Resource ReSerVation Protocol (RSVP) Version 1 Functional Specification," RFC 2205, Sept. 1997.
[2] J. Wroclawsky, "The Use of RSVP with IETF Integrated Services," RFC 2210, Sept. 1997.

[3] G. Bianchi, N. Blefari-Melazzi, and M. Femminella "A Migration Path to provide End-to-End QoS over Stateless Networks by Means of a Probing-Driven Admission Control," Internet draft, draft-bianchi blefari-end-toend-QoS-00.txt, Dec. 2000.

[4] D. B. Johnson and C. Perkins; "Mobility Support in IPv6"; Internet draft, draft-ietf-mobileip-ipv6-12.txt, Apr. 27, 2000, work in progress.

[5] A. De Carolis, "QoS-Aware Handover for Mobile IP: Secondary Home Agent," Internet draft, draft-decarolisqoshandover-02.txt, Apr. 2000.

[6] J. T. Malinen and C. E. Perkins, "Mobile IPv6 Regional Registration," Internet draft, draft-malinen-mobileipregreg6-00.txt, July 2000.

[7] C. Castelluccia, "A Hierarchical Mobile IPv6 Proposal," INRIA Rapport Technique no. 0226, Nov. 1998.

[8] J. Bound, M. Carney, and C. Perkins, "Dynamic Host Configuration Protocol for IPv6 (DHCPv6)," Internet draft, draftietf-dhc-dhcpv6-15.txt, May 5, 2000; work in progress.

\section{AdDitiOnAL READING}

[1] G. Huston, "Interconnection, Peering and Settlements," IP J., vol. 2, no. 1, Mar. 1999.

[2] S. Blake, "An Architecture for Differentiated Services," RFC 2475, Dec. 1998

\section{BIOGRAPHIES}

PAOLO CONFORTO (p.conforto@roma.alespazio.it) received an M.S. degree in electronic engineering from the University of Rome "La Sapienza" in 1998. In 1998 he received a postdegree grant from the University of Rome "La Sapienza" Dipartimento di Informatica e Sistemistica and worked in the scope of several Advanced Communication Technologies and Services (ACTS) projects funded by the European Commission. Since March 1999 he has worked at Alenia Spazio where he is currently involved in the Information Society Technologies (IST) SUITED project sponsored by the European Commission. Since March 2000 he is also a Ph.D. candidate at the University of Rome "La Sapienza" Dipartimento di Informatica e Sistemistica. His research interests 
mainly focus on the integration of satellite systems with wireless and fixed IP-based networks.

CLEMENTINA TOCCI received a summa cum laude M.Sc. degree in electronic engineering from the University of Rome "La Sapienza" in 1999, with a final thesis on integration of ATM-based multimedia access networks, developed in the framework of the ACTS project ACCORD. In 1999 she received a post-degree grant issued by the Dipartimento di Informatica e Sistemistica, University of Rome "La Sapienza," and continued working on the ACTS project "ACCORD" in synergy with Alenia Spazio. Since October 1999 she has been with Alenia Spazio and is currently involved in the system design of a global mobile broadband system for the provision of IP-based mobile and QoS-sensitive services developed in the framework of IST project SUITED.

GIACINTO LOSQUADRO received a summa cum laude M.S. in electronic engineering from the University of Pisa in the 1977. He joined Telettra SpA in 1979, as design engineer in the field of high-bit-rate transmissions and regeneration. Since 1980, with Alenia Spazio SpA, he has been project leader of several national and international projects for space communications and remote sensing, like: the SICRAL Italian military satellite system, the Data Relay Satellite component of the ESA Artemis mission, the ESA ERS1 radar altimeter, the ITALSAT multibeam payload, the ESA Huygens Probe Data Relay, and ESA On Board Processing projects. Since 1997 he is responsible for the Network Engineering Unit of Alenia Spazio SpA, where he manages the development of the EuroSkyWay satellite system and related $R \& D$ activities, including many influential satellite communications projects, principally funded under the EC and ESA R\&D programs. He holds five international patents, and is a member of the IEE's Technical Advisory Panel for Satellite Networks.
RAY E. SHERIFF graduated from the University of Leeds, United Kingdom, in 1986. He spent the next four and half years in the satellite communications industry. In 1991 he took up a lectureship with the University of Bradford, United Kingdom, where he was awarded a Ph.D. in 1995 and appointed reader in mobile communications in 2000. During his time at Bradford, he has participated in a number of influential satellite communications projects, principally funded under the EC's R \& D programs. He is an Executive Committee member of the IEE's Satellite Systems and Applications Professional Network and a U.K. Delegate on the Management Committee of the COST 272 Action.

PAULINE M. L. CHAN received an electronic systems and information engineering degree from Sheffield Hallam University United Kingdom, in 1999 following the award of a JPA Scholarship from the Malaysian Government. She is currently in the final year of her Ph.D. at the University of Bradford, United Kingdom, where she is researching on the design of satellite network procedures for heterogeneous packet-oriented environments. She participates in the EC's IST SUITED project and was previously involved in the COST 253 Action.

Y. Fun Hu received a 1 st Class B.Sc. degree in mathematical sciences and a Ph.D. degree in information systems engineering, both from the University of Bradford. In 1990 she joined the University of Leeds, United Kingdom, as a research fellow after working two years in the satellite communications industry. In 1994 she was appointed lecturer at the University of Bradford. She is now a senior lecturer in the same university. She has been involved in several defense, ESA, and EU funded projects, including SAINT, SINUS, SUMO, VANTAGE, SECOMS, and SUITED. She was a national delegate to the EU COST 253 and COST 256 Actions. She is now a national delegate to the EU COST 272 Action and a member of the Executive Committee of the IEE Satellite Systems and Applications Professional Network.
An extensive

experimental

campaign is

envisaged in the

framework of the

SUITED project to

test and validate

the main func-

tionality devised

for the GMBS

system for the

support of both

mobility and QoS. 